\title{
Prevalence and molecular characterization of Vibrio cholerae from fruits and salad vegetables sold in Jakarta, Indonesia, using most probable number and PCR
}

\author{
Andrea Budiman, Kevin Kurnia and Diana E. Waturangi* (1)
}

\begin{abstract}
Objective: Cholera is an intestinal infection caused by Vibrio cholerae, it is usually occurs in developing countries that lack of sanitation. In developing country including Indonesia, awareness importance of sanitation is still low. Unfortunately, research related to the detection of $V$. cholerae from fruit and vegetables in Indonesia is still rare. In this study, MPN method was used to determine the prevalence of $V$. cholerae followed by single and multiplex PCR to detect virulence genes, including toxR, ctxA, tcpA, hlyA, ace, ompU, and zot.

Results: We found 3 fruits and 2 vegetables positive for tox $R$ gene. Fruit samples which were showed tox $R$ positive found from East Jakarta while for vegetables, it was recovered from West Jakarta and Central Jakarta. Twenty-three isolates were recovered from tox $R$ positive samples. The result of antibiotic resistance analysis showed that $4.35 \%$ of the isolates resistant to gentamicin, streptomycin (17.39\%), trimethoprim (52.17\%), ciprofloxacin (30.43\%), ampicillin (13.04\%), nalidixic acid (82.61\%), and polymyxin B (91.30\%). None of these isolates were resistant to kanamycin. Combination of MPN and Multiplex PCR method can be used to detect the prevalence and characterize the virulence properties of $V$. cholerae.
\end{abstract}

Keywords: Vibrio cholerae, Fruits, Vegetables, MPN, Multiplex PCR

\section{Introduction}

Cholera is an infection in the small intestine caused by Vibrio cholerae. The main symptoms of cholera are vomiting and diarrhea that lead to dehydration and electrolyte imbalance. According to WHO (2015) [1], the incidence rate of cholera in Jakarta, Indonesia reached 0.45 cases per 1,000 populations with a case fatality rate of $1 \%$. In 1961, $V$. cholerae O1 biotype El Tor was emerged in Celebes (Sulawesi), Indonesia and spread to the other islands of Indonesia and other countries causing the seventh pandemic of cholerae. While the first six of cholera

*Correspondence: diana.waturangi@atmajaya.ac.id

Atma Jaya Catholic University of Indonesia, Faculty of Biotechnology,

Jalan Raya Cisauk-Lapan No. 10, 15345 Tangerang, Banten, Indonesia pandemics in 1899-1923 were caused by the classical strains [2]. Cholera is the second leading cause of death for children under the age of 5 and at least 120,000 deaths occurred each year [3]. $V$. cholerae is a Gram-negative bacteria, facultative anaerobe, oxidase-positive, and have a single polar flagellum [4].

In small intestine, $V$. cholerae produces enterotoxins to disrupt ion transport and lead to dehydration [5]. The toxin production depends on the transcriptional activator ToxR that regulate cholera toxin gene expression (ctxA), TCP biogenesis (tcpA), outer membrane protein expression (ompU), and at least 17 distinct genes in $\mathrm{O} 139$ and $\mathrm{O} 1$ strains $[6,7]$. There are many virulence factors that can cause cholera with different characteristics aside from cholera toxin, such as El Tor-like hemolysin (hlyA), 
a new CT, outer membrane protein (ompU), Shiga-like toxin (stx), and a zonula occludens toxin (zot) [8].

Conventional methods used to detect and characterize $V$. cholerae from samples are time-consuming, low sensitivity, and laborious. Furthermore, these methods are not efficient for the screening of a large number of samples [9]. Therefore, molecular methods are required for rapid and sensitive detection of $V$. cholerae by using specific primers associated with virulence genes. The objective of this research is to provide rapid and reliable method of detection of $V$. cholerae from salad vegetables and fruits. Also, to determine the prevalence and detection of virulence genes of $V$. cholerae.

\section{Main text \\ Methods \\ Samples collection and handling}

A total of 133 samples (57 fruits and 76 vegetables) were collected from several traditional markets and supermarkets in Jakarta from July 2012 until March 2013. We collected 10 fruits and 10 vegetables for each districts (North, South, West, East, and Central of Jakarta) to represent the data for Jakarta region. Fruits that could be eaten without peeling and vegetables that often eaten raw were selected for this study. Apple-Malus domestica, Star fruit-Averrhoa carambola, Rose apple-Syzygium jambos, Guava-Psidium guajava, Pear-Pyrus L., Tomato-Solanum lycopersicum, Grape-Vitis vinifera, Bean sprout_-Vigna radiata, Black nightshade-Solanum nigrum, Cabbage-Brassica oleracea, Carrot-Daucus carota, Chayote-Sechium edule, Coriander-Coriandrum sativum, Cucumber-Cucumis sativus, Lemon basil-Ocimum citriodorum, Lettuce-Lactuca sativa, Thai eggplant-Solanum melongena, Pea Shoot-Pisum sativum, Ulam raja-Cosmos caudatus, WatercressNasturtium officinale, Yard longbean-Vigna unguiculata. Samples were processed maximum $24 \mathrm{~h}$ after purchasing from the vendors.

\section{Bacteria enumeration from samples}

From each samples, about $5 \mathrm{~g}$ of cortex were cut and crushed. Then, it were inoculated to $25 \mathrm{ml}$ of Alkaline Peptone Water (APW) medium pH 8.5 (Oxoid, England) and vortexed for $1 \mathrm{~min}$. For enumeration, we used 3 tube of Most Probable Number (MPN) method. There were 9 tubes for each samples with dilution of $10^{-1}, 10^{-2}$, and $10^{-3}$ which were divided equally. Nine MPN tubes of $10^{-1}$ dilution were added into $1 \mathrm{ml}$ of solutions and vortexed for $1 \mathrm{~min}$. Subsequently, $1 \mathrm{ml}$ of samples were taken from $10^{-1}$ dilution and mixed with $10^{-2}$ dilution and so on until reached $10^{-3}$ dilution [6]. MPN tubes were incubated at V. cholerae optimum condition $37^{\circ} \mathrm{C}$ and $120 \mathrm{rpm}$ for $24 \mathrm{~h}$.

\section{DNA templates preparation}

DNA template for single PCR was prepared from positive MPN tubes. One $\mathrm{ml}$ of the cultures were centrifuged $12,000 \times g$ for $5 \mathrm{~min}$. Then, the supernatant was taken and resuspended in $200 \mu \mathrm{l}$ of ddH2O, and boiled for $10 \mathrm{~min} \mathrm{[10].} \mathrm{The} \mathrm{Supernatant} \mathrm{was} \mathrm{used} \mathrm{as} \mathrm{DNA}$ template and stored at $-20^{\circ} \mathrm{C}$ until further used.

\section{Uniplex and Multiplex PCR}

Uniplex PCR were used to detect the presence of toxR gene [11] from $V$. cholerae suspected samples. V. cholerae 3.21 (Atma Jaya culture collection) were used as positive control. The Total of PCR reaction was $25 \mu \mathrm{l}$ containing of $12.5 \mu \mathrm{l}$ of GoTaq green master mix (400 $\mu \mathrm{M}$ dNTP) (Promega, USA), $1 \mu \mathrm{l}(10 \mu \mathrm{M})$ of each tox $R$ forward and reverse primers, $2 \mu$ of DNA template, and $8.5 \mu \mathrm{l}$ of nuclease free water. PCR reaction was performed in a thermal cycler (Applied Biosystems, USA) with initial denaturation at $94{ }^{\circ} \mathrm{C}$ for $2 \mathrm{~min}$, followed by 25 cycles consisting of $94{ }^{\circ} \mathrm{C}$ for $1 \mathrm{~min}$, $62{ }^{\circ} \mathrm{C}$ for $1 \mathrm{~min}$, and $72{ }^{\circ} \mathrm{C}$ for $1 \mathrm{~min}$, and a post-extension step $72{ }^{\circ} \mathrm{C}$ for $10 \mathrm{~min}$.

Multiplex PCR were used to detect other virulenceassociated genes such as, $c t x A, t c p A, h l y A$, ace, ompU, and $z o t[8,11]$. The PCR mixture consisted of $25 \mu \mathrm{l}$ of GoTaq green master mix, $1 \mu \mathrm{l}$ of each primer pairs, $2.5 \mu \mathrm{l}$ of DNA template, and nuclease free water until $50 \mu \mathrm{l}$ of mixture. The final concentration used for the toxR primer was $50 \mu \mathrm{M} ; 16 \mu \mathrm{M}$ for $\operatorname{ctx} A$ and ompU primer; $30 \mu \mathrm{M}$ for $z o t$, ace, hlyA, and tcpA primer. PCR condition were initial denaturation at $94{ }^{\circ} \mathrm{C}$ for $2 \mathrm{~min}$ followed by 20 cycles consisting of $94^{\circ} \mathrm{C}$ for $1 \mathrm{~min}$, $62{ }^{\circ} \mathrm{C}$ for $1 \mathrm{~min}$, and $72{ }^{\circ} \mathrm{C}$ for $1 \mathrm{~min}, 10$ cycles consisting of $94{ }^{\circ} \mathrm{C}$ for $1 \mathrm{~min}, 54{ }^{\circ} \mathrm{C}$ for $1 \mathrm{~min}$, and $72{ }^{\circ} \mathrm{C}$ for $1 \mathrm{~min}$, then post-extension step $72{ }^{\circ} \mathrm{C}$ for $10 \mathrm{~min}$, and hold at $4{ }^{\circ} \mathrm{C}$.

The PCR products were loaded on $1 \%(\mathrm{w} / \mathrm{v})$ agarose gel with $1 \times$ Tris Acetic EDTA (TAE) buffer and run with condition of $60 \mathrm{~V}$ for $150 \mathrm{~min}$, and visualized under UV light after stained with ethidium bromide (EtBr).

\section{Isolation of V. cholerae suspect}

All of $V$. cholerae suspected colonies were selected from Thiosulphate Citrate Bile-salt Sucrose (TCBS) agar and grown at BHIA medium overnight. These colonies were tested with oxidase reagent $1 \%(\mathrm{w} / \mathrm{v})$ solution of NNNN Tetra methyl-p-Phenylendiammonium dichloride (Merck). V. cholerae sh positive result (form purple colour after $30 \mathrm{~s}$ ) for oxidase test on filter paper [6]. Afterwards, fresh positive culture from agar were taken with toothpick and stirred with $200 \mu \mathrm{lddH} 2 \mathrm{O}$ until homogenized. 
Table 1 MPN results and presence of toxR gene for $V$. cholerae isolates from Jakarta, Indonesia region

\begin{tabular}{|c|c|c|c|c|c|c|}
\hline \multirow[t]{2}{*}{ Sample type } & \multirow[t]{2}{*}{ Source } & \multirow[t]{2}{*}{ Sample ID } & \multicolumn{2}{|c|}{$\begin{array}{l}\text { V. cholerae level } \\
\text { (MPN/ml) } \\
95 \% \text { confidence } \\
\text { limit }\end{array}$} & \multirow[t]{2}{*}{ toxR result by PCR } & \multirow[t]{2}{*}{$\begin{array}{l}\text { \% toxR } \\
\text { positive } \\
\text { samples }\end{array}$} \\
\hline & & & Lower & Upper & & \\
\hline \multirow[t]{36}{*}{ Fruits } & North Jakarta & TO.U.1 & 420 & - & - & 0 \\
\hline & & TO.U.2 & 42 & 1000 & - & \\
\hline & & AP.U.1 & 9 & 180 & - & \\
\hline & & AP.U.2 & 42 & 1000 & - & \\
\hline & & JB.U.1 & 42 & 1000 & - & \\
\hline & & JA.U.1 & 420 & - & - & \\
\hline & & PI.U.1 & 42 & 1000 & - & \\
\hline & & TO.U.3 & 420 & - & - & \\
\hline & & BL.U.1 & 420 & - & - & \\
\hline & & TO.U.4 & 420 & - & - & \\
\hline & South Jakarta & BL.S.1 & 420 & - & - & 0 \\
\hline & & TO.S.1 & 180 & 4100 & - & \\
\hline & & PI.S.1 & - & 9.5 & - & \\
\hline & & TO.S.2 & 18 & 420 & - & \\
\hline & & BL.S.2 & 9 & 180 & - & \\
\hline & & JA.S.1 & 420 & - & - & \\
\hline & & JB.S.1 & 420 & - & - & \\
\hline & & BL.S.3 & 420 & - & - & \\
\hline & & TO.S.3 & 420 & - & - & \\
\hline & & AP.S.1 & 18 & 420 & - & \\
\hline & & PI.S.2 & 18 & 420 & - & \\
\hline & & TO.S.4 & 420 & - & - & \\
\hline & West Jakarta & AP.B.1 & 18 & 420 & - & 0 \\
\hline & & BL.B.1 & 420 & - & - & \\
\hline & & JA.B.1 & 420 & - & - & \\
\hline & & JB.B.1 & 420 & - & - & \\
\hline & & TO.B.1 & 420 & - & - & \\
\hline & & TO.B.2 & 420 & - & - & \\
\hline & & JB.B.2 & 420 & - & - & \\
\hline & & TO.B.3 & 420 & - & - & \\
\hline & & BL.B.2 & 420 & - & - & \\
\hline & & TO.B.4 & 420 & - & - & \\
\hline & & PI.B.1 & 180 & 4100 & - & \\
\hline & East Jakarta & JA.T.1 & 420 & - & - & 21.4 \\
\hline & & TO.T.1 & 420 & - & - & \\
\hline & & BL.T.1 & 420 & - & - & \\
\hline
\end{tabular}


Table 1 (continued)

\begin{tabular}{|c|c|c|c|c|c|c|}
\hline \multirow[t]{2}{*}{ Sample type } & \multirow[t]{2}{*}{ Source } & \multirow[t]{2}{*}{ Sample ID } & \multicolumn{2}{|c|}{$\begin{array}{l}\text { V. cholerae level } \\
\text { (MPN/ml) } \\
95 \% \text { confidence } \\
\text { limit }\end{array}$} & \multirow[t]{2}{*}{ toxR result by PCR } & \multirow[t]{2}{*}{$\begin{array}{l}\% \text { toxR } \\
\text { positive } \\
\text { samples }\end{array}$} \\
\hline & & & Lower & Upper & & \\
\hline & & PI.T.1 & 420 & - & - & \\
\hline & & AP.T.1 & 42 & 1000 & - & \\
\hline & & BL.T.2 & 180 & 4100 & - & \\
\hline & & JB.T.1 & 180 & 4100 & - & \\
\hline & & PI.T.2 & 42 & 1000 & - & \\
\hline & & AG.T.1 & 180 & 4100 & - & \\
\hline & & AP.T.2 & 420 & - & + & \\
\hline & & BL.T.3 & 420 & - & - & \\
\hline & & PI.T.3 & 420 & - & + & \\
\hline & & JB.T.2 & 420 & - & - & \\
\hline & & BL.T.4 & 420 & - & + & \\
\hline & Central Jakarta & TO.P.1 & 420 & - & - & 0 \\
\hline & & TO.P.2 & 420 & - & - & \\
\hline & & AP.P.1 & 420 & - & - & \\
\hline & & AP.P.2 & 4.6 & 94 & - & \\
\hline & & PI.P.1 & 180 & 4100 & - & \\
\hline & & TO.P.3 & 420 & - & - & \\
\hline & & TO.P.4 & 420 & - & - & \\
\hline & & AP.P.3 & 42 & 1000 & - & \\
\hline & & BL.P.1 & 420 & - & - & \\
\hline & & TO.P.5 & 420 & - & - & \\
\hline \multirow[t]{17}{*}{ Vegetables } & North Jakarta & SEL.U.1 & 420 & - & - & 0 \\
\hline & & KOL.U.1 & 420 & - & - & \\
\hline & & KET.U.1 & 420 & - & - & \\
\hline & & KER.U.1 & 420 & - & - & \\
\hline & & KEM.U.1 & 420 & - & - & \\
\hline & & SEA.U.1 & 420 & - & - & \\
\hline & & KET.U.2 & 420 & - & - & \\
\hline & & WOR.U.1 & 420 & - & - & \\
\hline & & KOL.U.2 & 4.6 & 94 & - & \\
\hline & & KOL.U.3 & 420 & - & - & \\
\hline & & KET.U.3 & 420 & - & - & \\
\hline & & WOR.U.2 & 420 & - & - & \\
\hline & South Jakarta & SEL.S.1 & 420 & - & - & 0 \\
\hline & & SEA.S.1 & 420 & - & - & \\
\hline & & KET.S.1 & 420 & - & - & \\
\hline & & KOL.S.1 & 420 & - & - & \\
\hline & & SEL.S.2 & 420 & - & - & \\
\hline
\end{tabular}


Table 1 (continued)

\begin{tabular}{|c|c|c|c|c|c|c|}
\hline \multirow[t]{2}{*}{ Sample type } & \multirow[t]{2}{*}{ Source } & \multirow[t]{2}{*}{ Sample ID } & \multicolumn{2}{|c|}{$\begin{array}{l}\text { V. cholerae level } \\
\text { (MPN/ml) } \\
95 \% \text { confidence } \\
\text { limit }\end{array}$} & \multirow[t]{2}{*}{ toxR result by PCR } & \multirow[t]{2}{*}{$\begin{array}{l}\% \text { toxR } \\
\text { positive } \\
\text { samples }\end{array}$} \\
\hline & & & Lower & Upper & & \\
\hline & & KET.S.2 & 420 & - & - & \\
\hline & & KOL.S.2 & 420 & - & - & \\
\hline & & SEL.S.3 & 420 & - & - & \\
\hline & & KET.S.3 & 420 & - & - & \\
\hline & & KOL.S.3 & 420 & - & - & \\
\hline & & SEL.S.4 & 420 & - & - & \\
\hline & & KET.S.4 & 420 & - & - & \\
\hline & & WOR.S.1 & 420 & - & - & \\
\hline & & ULA.S.1 & 420 & - & - & \\
\hline & & SEA.S.1 & 420 & - & - & \\
\hline & & SEL.S.5 & 420 & - & - & \\
\hline & & KEM.S.1 & 420 & - & - & \\
\hline & & KOL.S.4 & 420 & - & - & \\
\hline & West Jakarta & KOL.B.1 & 420 & - & - & 5.26 \\
\hline & & KOL.B.2 & 420 & - & - & \\
\hline & & KET.B.1 & 420 & - & - & \\
\hline & & SEL.B.1 & 420 & - & - & \\
\hline & & KOL.B.3 & 420 & - & - & \\
\hline & & KOL.B.4 & 420 & - & - & \\
\hline & & KET.B.2 & 420 & - & - & \\
\hline & & SEL.B.2 & 420 & - & - & \\
\hline & & WOR.B.1 & 420 & - & - & \\
\hline & & DOU.B.1 & 420 & - & - & \\
\hline & & SEL.B.3 & 420 & - & - & \\
\hline & & LAB.B.1 & 420 & - & - & \\
\hline & & KET.B.3 & 420 & - & - & \\
\hline & & KOL.B.5 & 420 & - & - & \\
\hline & & WOR.B.2 & 420 & - & + & \\
\hline & & KET.B.4 & 420 & - & - & \\
\hline & & TAU.B.1 & 420 & - & - & \\
\hline & & WOR.B.3 & 420 & - & - & \\
\hline & & KOL.B.6 & 420 & - & - & \\
\hline & East Jakarta & SEL.T.1 & 420 & -- & - & 0 \\
\hline & & KEM.T.1 & 420 & - & - & \\
\hline & & KOL.T.1 & 420 & - & - & \\
\hline & & KEM.T.2 & 420 & - & - & \\
\hline & & SEL.T.2 & 420 & - & - & \\
\hline & & TER.T.1 & 420 & - & - & \\
\hline & & KET.T.1 & 420 & - & - & \\
\hline & & TER.T.2 & 420 & - & - & \\
\hline
\end{tabular}


Table 1 (continued)

\begin{tabular}{|c|c|c|c|c|c|c|}
\hline \multirow[t]{2}{*}{ Sample type } & \multirow[t]{2}{*}{ Source } & \multirow[t]{2}{*}{ Sample ID } & \multicolumn{2}{|c|}{$\begin{array}{l}\text { V. cholerae level } \\
\text { (MPN/ml) } \\
95 \% \text { confidence } \\
\text { limit }\end{array}$} & \multirow[t]{2}{*}{ toxR result by PCR } & \multirow[t]{2}{*}{$\begin{array}{l}\text { \% toxR } \\
\text { positive } \\
\text { samples }\end{array}$} \\
\hline & & & Lower & Upper & & \\
\hline & & LEU.T.1 & 420 & - & - & \\
\hline & & TER.T.3 & 420 & - & - & \\
\hline & & KET.T.2 & 420 & - & - & \\
\hline & \multirow[t]{16}{*}{ Central Jakarta } & KAC.P.1 & 420 & - & - & 6.25 \\
\hline & & KEM.P.1 & 420 & - & - & \\
\hline & & TER.P.1 & 420 & - & - & \\
\hline & & KEM.P.2 & 420 & - & - & \\
\hline & & KET.P.1 & 420 & - & - & \\
\hline & & TAU.P.1 & 420 & - & - & \\
\hline & & KOL.P.1 & 420 & - & - & \\
\hline & & WOR.P.1 & 420 & - & - & \\
\hline & & SEL.P.1 & 8.7 & 94 & + & \\
\hline & & KOL.P.2 & 420 & - & - & \\
\hline & & KOL.P.3 & 420 & - & - & \\
\hline & & WOR.P.2 & 420 & - & - & \\
\hline & & SEL.P.2 & 420 & - & - & \\
\hline & & KET.P.2 & 420 & - & - & \\
\hline & & KET.P.3 & 420 & - & - & \\
\hline & & KOL.P.4 & 420 & - & - & \\
\hline
\end{tabular}

AP Apple, BL Star fruit, JA Rose apple, JB Guava, PI Pear, TO Tomato, AG Grape, TAU Bean sprout, LEU Black nightshade, KOL Cabbage, WOR Carrot, LAB Chayote, KER Coriander, KET Cucumber, KEM Lemon basil, SEL Lettuce, TER Thai eggplant, DOU Pea Shoot, ULA Ulam raja, SEA Watercress, KAC Yard longbean

\section{Serological and antibiotic resistance assay}

Recovered $V$. cholerae from samples were refreshed into BHIA medium and incubated at $37{ }^{\circ} \mathrm{C}$ overnight and continued with serological assay using antiserum polyvalent O1 (Remel) and monovalent (Ogawa or Inaba) [6]. Firstly, the isolate was tested with polyvalent antiserum, if the result showed positive for serogroup $\mathrm{O} 1$, it will tested further with monovalent antiserum to determine whether it was serotype of Ogawa or Inaba. $V$. cholerae which were showed positive reaction with antiserum will form white clumps when it react with antiserum.

For antibiotic resistance assay, $V$. cholerae isolates were streaked on nutrient agar (NA) and incubated overnight at $37{ }^{\circ} \mathrm{C}$. In this study, we used antibiotic discs such as polymyxin B (300 U), nalidixic acid $(30 \mu \mathrm{g})$, trimethoprim $(5 \mu \mathrm{g})$, ampicillin $(10 \mu \mathrm{g})$, streptomycin $(10 \mu \mathrm{g})$, gentamycin $(10 \mu \mathrm{g})$, kanamycin $(30 \mu \mathrm{g})$, and ciprofloxacin (5 $\mu \mathrm{g}$ ) (Oxoid, Hampshire, England). Zone of inhibition was determined followed the guideline from Clinical and Laboratory Standard Institute (CLSI) [12].

\section{Results}

A total of $99.25 \%$ of the samples (from 133 samples) showed positive results for MPN assay (Table 1). The prevalence rate of $V$. cholerae in fruits and vegetables obtained from 5 regions of Jakarta was $100 \%$, except for fruits collected from South Jakarta (91.67\%). A positive result means at least one MPN tube (out of 9 tubes) was turbid. The highest lower and upper number from MPN result was $420 \mathrm{MPN} / \mathrm{ml}$ and $>4100 \mathrm{MPN} / \mathrm{ml}$ respectively found in 36 samples fruits and 74 samples vegetables. The lowest lower and upper number was found from apple (Central Jakarta) and cabbage (North Jakarta) with value of $4.6 \mathrm{MPN} / \mathrm{ml}$ and $94 \mathrm{MPN} / \mathrm{ml}$, respectively. Moreover, 3 fruits and 2 vegetables were positive for toxR gene detection.

Total single colonies of $V$. cholerae recovered from positive samples were 23 colonies. These isolates recovered from apple (5), star-fruit (1), pear (7), carrot (2), and lettuce (8). Most of the isolates were found from East Jakarta (13 of 23; 56.52\%). None of fruits sample from Central and West Jakarta was positive, as well as for vegetables, no positive result found from East Jakarta. 
Table 2 V. cholerae recovered from fruits and vegetables samples based on their subtypes

\begin{tabular}{|c|c|c|c|c|c|c|c|c|c|c|c|c|}
\hline \multirow[t]{2}{*}{ No } & \multirow[t]{2}{*}{ Isolate } & \multirow[t]{2}{*}{ Source } & \multicolumn{2}{|l|}{ Antiserum } & \multirow{2}{*}{$\begin{array}{l}\text { Polymyxin B } \\
\text { Biotype }\end{array}$} & \multicolumn{7}{|c|}{ Multiplex PCR for virulence genes detection } \\
\hline & & & Poly-valent & Mono-valent & & tox $R$ & $\operatorname{ctx} A$ & $\operatorname{tcp} A$ & zot & ace & ompU & hlyA \\
\hline & $\begin{array}{l}\text { V. cholerae } 3.21 \\
\text { (+ control) }\end{array}$ & & Non-O1 & & & + & + & - & - & - & - & - \\
\hline 1 & WOR.B.2.1 & West Jakarta & 01 & Ogawa & El Tor & + & - & - & - & - & - & - \\
\hline 2 & WOR.B.2.2 & & 01 & Ogawa & Classical & + & - & - & - & - & - & - \\
\hline 3 & AP.T.2.1 & East Jakata & Non-O1 & & & + & - & - & - & - & - & - \\
\hline 4 & AP.T.2.2 & & 01 & Inaba & Classical & + & - & - & - & - & - & - \\
\hline 5 & AP.T.2.3 & & Non-O1 & & & + & - & - & - & - & - & - \\
\hline 6 & AP.T.2.4 & & Non-O1 & & & + & - & - & - & - & - & - \\
\hline 7 & AP.T.2.5 & & Non-O1 & & & + & - & - & - & - & - & - \\
\hline 8 & PI.T.3.1 & & $\mathrm{O} 1$ & Inaba & El Tor & + & - & - & - & - & - & - \\
\hline 9 & PI.T.3.2 & & 01 & Inaba & El Tor & + & - & - & - & - & - & - \\
\hline 10 & PI.T.3.3 & & 01 & Ogawa & El Tor & + & - & - & - & - & - & - \\
\hline 11 & PI.T.3.4 & & 01 & Inaba & El Tor & + & - & - & - & - & - & - \\
\hline 12 & PI.T.3.5 & & 01 & Inaba & El Tor & + & - & - & - & - & - & - \\
\hline 13 & PI.T.3.6 & & 01 & Inaba & El Tor & + & - & - & - & - & - & - \\
\hline 14 & PI.T.3.7 & & $\mathrm{O} 1$ & Ogawa & El Tor & + & - & - & - & - & - & - \\
\hline 15 & BL.T.4.1 & & Non-O1 & & & + & - & - & - & - & - & - \\
\hline 16 & SEL.P.1.1 & Central Jakarta & O1 & Ogawa & El Tor & + & - & - & - & - & - & - \\
\hline 17 & SEL.P.1.2 & & 01 & Inaba & El Tor & + & - & - & - & - & - & - \\
\hline 18 & SEL.P.1.3 & & 01 & Ogawa & ElTor & + & - & - & - & - & - & - \\
\hline 19 & SEL.P.1.4 & & $\mathrm{O} 1$ & Ogawa & El Tor & + & - & - & - & - & - & - \\
\hline 20 & SEL.P.1.5 & & 01 & Ogawa & ElTor & + & - & - & - & - & - & - \\
\hline 21 & SEL.P.1.6 & & $\mathrm{O} 1$ & Ogawa & ElTor & + & - & - & - & - & - & - \\
\hline 22 & SEL.P.1.7 & & $\mathrm{O} 1$ & Ogawa & ElTor & + & - & - & - & - & - & - \\
\hline 23 & SEL.P.1.8 & & 01 & Inaba & ElTor & + & - & - & - & - & - & - \\
\hline
\end{tabular}

AP Apple, BL Star fruit, PI Pear, WOR Carrot, SEL Lettuce

Most of the single isolates belong to the serogroup O1 ( 18 of $23 ; 78.26 \%$ ) with 10 isolates belong to Ogawa serotype and 8 serotype of Inaba (Table 2). Ogawa serotype is more prevalent compare with Inaba with the percentage respectively $55.56 \%$ and $44.44 \%$. While for El Tor biotype, was found more dominant compare with Classical with the percentage $88.89 \%$ for O1. All of the single isolates showed the presence of tox $R$ regulator genes, but we did not find other virulence genes associated genes using primer as describe in the method.

Antibiotic resistance assay showed that $4.35 \%$ of the isolates showed resistant to gentamicin, $17.39 \%$ resistant to streptomycin, $52.17 \%$ resistant to trimethoprim, $30.43 \%$ resistant to ciprofloxacin, $13.04 \%$ resistant to ampicillin, $82.61 \%$ resistant to nalidixic acid, and $91.30 \%$ resistant to polymyxin B. None of these isolates showed resistant to kanamycin (Table 3). Resistance to polymyxin $\mathrm{B}$ can be used to determine as biotype serogroup O1, since biotype El Tor has resistance trait for polymyxin B and otherwise for the Classical.

\section{Discussions}

In this study, we utilized the MPN method for rapid estimation of $V$. cholerae prevalence in samples. On the other hand TCBS as selective medium and oxidase test were also used to ensure the presence of $V$. cholerae. The confirmation was done through PCR to distinguish between the pathogenic and non-pathogenic $V$. cholerae through detection of virulence-associated genes. Combination of MPN and PCR assay gives us rapid and sensitive detection and quantification of the presence of $V$. cholerae in fruits and vegetables, compare with conventional MPN method only.

The presence of toxR gene is important for regulation of other virulence-associated genes in pathogenic $V$. cholerae [13]. Therefore, this gene is used mainly to detect presence of pathogenic $V$. cholerae from samples. Pathogenic $V$. cholerae was detected in fruits from East Jakarta (21.4\%) and vegetables from West Jakarta (5.26\%) and Central Jakarta (6.25\%). The difference in prevalence number might happened due to the distribution 
Table 3 Antibiotic resistance assay of $V$. cholerae

\begin{tabular}{|c|c|c|c|c|c|c|c|c|c|c|}
\hline \multirow[t]{2}{*}{ No } & \multirow[t]{2}{*}{ Isolates } & \multirow[t]{2}{*}{ Source } & \multicolumn{8}{|c|}{ Antibiotic Disc Assay } \\
\hline & & & $\mathrm{CN}$ & $S$ & $\mathrm{~K}$ & w & CIP & AMP & NA & PB \\
\hline 1 & WOR.B.2.1 & West Jakarta & $S$ & S & $S$ & $\mathrm{R}$ & $S$ & s & $\mathrm{R}$ & $\mathrm{R}$ \\
\hline 2 & WOR.B.2.2 & & $S$ & S & $\mathrm{S}$ & $R$ & $\mathrm{R}$ & S & $\mathrm{R}$ & S \\
\hline 3 & AP.T.2.1 & East Jakata & $S$ & $S$ & $\mathrm{~S}$ & $S$ & $S$ & s & $\mathrm{R}$ & $\mathrm{R}$ \\
\hline 4 & AP.T.2.2 & & $S$ & $S$ & $\mathrm{~S}$ & $S$ & $S$ & S & $\mathrm{R}$ & S \\
\hline 5 & AP.T.2.3 & & $S$ & $\mathrm{~S}$ & S & S & S & S & $\mathrm{R}$ & $\mathrm{R}$ \\
\hline 6 & AP.T.2.4 & & $S$ & $\mathrm{~S}$ & S & S & S & S & $\mathrm{R}$ & $\mathrm{R}$ \\
\hline 7 & AP.T.2.5 & & $S$ & $S$ & S & S & $S$ & S & $\mathrm{R}$ & $\mathrm{R}$ \\
\hline 8 & PI.T.3.1 & & $S$ & $\mathrm{R}$ & $S$ & $S$ & $S$ & $R$ & $\mathrm{R}$ & $\mathrm{R}$ \\
\hline 9 & PI.T.3.2 & & $S$ & $S$ & $\mathrm{~S}$ & $S$ & $S$ & S & $\mathrm{R}$ & $\mathrm{R}$ \\
\hline 10 & PI.T.3.3 & & $S$ & $\mathrm{R}$ & $\mathrm{S}$ & $S$ & $S$ & $\mathrm{R}$ & $\mathrm{R}$ & $\mathrm{R}$ \\
\hline 11 & PI.T.3.4 & & S & $\mathrm{R}$ & S & S & S & R & $\mathrm{R}$ & $\mathrm{R}$ \\
\hline 12 & PI.T.3.5 & & $\mathrm{S}$ & $\mathrm{S}$ & S & $\mathrm{R}$ & S & S & $\mathrm{R}$ & $\mathrm{R}$ \\
\hline 13 & PI.T.3.6 & & $S$ & $\mathrm{~S}$ & S & $\mathrm{R}$ & S & S & $\mathrm{R}$ & $\mathrm{R}$ \\
\hline 14 & PI.T.3.7 & & $S$ & $\mathrm{R}$ & S & S & $S$ & S & $\mathrm{R}$ & $\mathrm{R}$ \\
\hline 15 & BL.T.4.1 & & $S$ & S & $\mathrm{S}$ & $R$ & $S$ & S & $\mathrm{R}$ & $\mathrm{R}$ \\
\hline 16 & SEL.P.1.1 & Central Jakarta & $\mathrm{S}$ & $\mathrm{S}$ & S & S & $\mathrm{R}$ & S & $\mathrm{R}$ & $\mathrm{R}$ \\
\hline 17 & SEL.P.1.2 & & $\mathrm{R}$ & $S$ & $\mathrm{~S}$ & $\mathrm{R}$ & $S$ & S & S & $\mathrm{R}$ \\
\hline 18 & SEL.P.1.3 & & $\mathrm{S}$ & $\mathrm{S}$ & S & $\mathrm{R}$ & $\mathrm{R}$ & S & $\mathrm{R}$ & $\mathrm{R}$ \\
\hline 19 & SEL.P.1.4 & & S & $\mathrm{S}$ & S & $\mathrm{R}$ & $\mathrm{R}$ & S & $\mathrm{R}$ & $\mathrm{R}$ \\
\hline 20 & SEL.P.1.5 & & $\mathrm{S}$ & $\mathrm{S}$ & S & $\mathrm{R}$ & $\mathrm{R}$ & S & $\mathrm{R}$ & $\mathrm{R}$ \\
\hline 21 & SEL.P.1.6 & & $S$ & $S$ & S & $R$ & $\mathrm{R}$ & S & S & $\mathrm{R}$ \\
\hline 22 & SEL.P.1.7 & & $S$ & $S$ & $S$ & $R$ & $\mathrm{R}$ & S & S & $R$ \\
\hline 23 & SEL.P.1.8 & & $S$ & $S$ & $S$ & $\mathrm{R}$ & $S$ & S & S & $\mathrm{R}$ \\
\hline
\end{tabular}

CN Gentamicin $(10 \mu \mathrm{g}), \mathrm{S}$ Streptomycin $(10 \mu \mathrm{g}), K$ Kanamycin $(30 \mu \mathrm{g})$, W Trimethoprim $(5 \mu \mathrm{g})$, CIP Ciprofloxacin $(5 \mu \mathrm{g})$, AMP Ampicillin $(10 \mu \mathrm{g}), N A$ Nalidixic acid $(30 \mu \mathrm{g})$, $P B$ Polymyxin B (300 U), R Resistant, S Susceptible

conditions, storage facilities, as well as sanitation in each market. In addition, some markets are located in poor and highly populated areas which is lack of access to clean water and proper sanitation. Cross-contamination may also occur since the wet market sells different types of raw meat and seafood.

For Ogawa serotype, with the percentage (55.56\%) showed higher than Inaba serotype (44.44\%). This results were in line with other publication found that Ogawa serotype was more prevalent than Inaba in Jakarta environment [14]. As the result of biotyping, El Tor biotype with the percentage $(88.89 \%)$ was found more dominant compare with Classical biotype (11.11\%). V. cholerae O1 El Tor is a biotype that responsible for the seventh pandemic in Celebes once.

Most $V$. cholerae found in this study belong to serogroup $\mathrm{O} 1$ which is known as the most often serogroup cause cholerae disease. Nevertheless, the non-O1 strain has also been reported to be involved in the emergence of a newer variant of $V$. cholerae $\mathrm{O} 139$ resulting in epidemic and pandemic [15]. Although non-O1 and O1 strains that we found, only had toxR genes, they still can cause cholera and mild gastroenteritis with unknown mechanisms [16], and there is also possibility that they might have other virulence-associated genes that had not been detected in this study Also, there is a possibility that non-pathogenic $V$. cholerae can evolve to pathogenic type due to the high possibility of horizontal virulence genes transfer [17].

\section{Conclusion}

Combination of MPN and Multiplex PCR methods are consider sufficient to assess the prevalence and detect the presence of virulence properties of $V$. cholerae. All of $V$. cholerae found from fruits and vegetables samples were considered harmless because of the absence of virulence-associated genes. But these isolates can not be denied as they still can evoke diarrhea with unknown mechanisms or they may have other virulence-associated genes that had not been detected in this research. On the other hand the presence of $V$. cholerae in fruits and vegetables showed improper sanitation of these products. Further research was also needed to to regularly study the prevalence and detect virulence-associated 
genes to reduce including prevention action to reduce the presence of non-O1 and $\mathrm{O} 1$ serogroup and its connection to diarrheal diseases outbreaks in Indonesia.

\section{Limitations}

The MPN method that was used in this study to enumerate Vibrio cholerae, might also detect other vibrio species. There is also a chance that few non-vibrio organisms are present in the enrichment medium.

\section{Abbreviations \\ MPN: Most probable number; CT: Cholera toxin; Omp: Outer membrane protein; Zot: Zonula occludens toxin.}

\section{Acknowledgements}

The author would also like to thanks for the advice from Prof. Swapan Banerjee Bureau of Microbial Hazards, HPFB, Health Canada / Government of Canada.

\section{Authors' contributions}

$\mathrm{AB}$ and $\mathrm{KK}$ conduct research, data analysis, and manuscript preparation. While DEW is a personal investigator and designing the proposal and advisory the research. All authors read and approved the final manuscript.

\section{Funding}

This research was supported by a grant to Dr. Diana E. Waturangi, M.Si from The International Foundation for Science (IFS) 2012.

\section{Availability of data and materials}

The datasets used and/or analysed during the current study are available from the corresponding author on reasonable request.

\section{Declarations}

Ethics approval and consent to participate

Not applicable.

\section{Consent for publication}

Not applicable.

\section{Competing interests}

The authors declare that they have no competing interests.

Received: 29 April 2021 Accepted: 4 February 2022

Published online: 03 March 2022

\section{References}

1. Ali M, Nelson AR, Lopez AL, Sack DA. Updated global burden of cholera in endemic countries. PLoS Negl Trop Dis. 2015;9(6):e0003832.

2. Kitaoka M, Miyata ST, Unterweger D, Pukatzki S. Antibiotic resistance mechanisms of Vibrio cholerae. J Med Microbiol. 2011;60:397-407.

3. Nelson EJ, Harris JB, Morris JG, Calderwood SB, Camilli A. Cholera transmission: the host, pathogen and bacteriophage dynamic. Nat Rev Microbiol. 2009;7:693-702.

4. Singh DV, Matte MH, Matte GR, Jiang S, et al. Molecular analysis of Vibrio cholerae 01, 0139, non-01, and non-0139 strains: clonal relationships between clinical and environmental isolates. Appl Environ Microbiol. 2001;67:910-21.

5. Maheswari M, Nelapati K, Kiranmayi B. Vibrio cholerae. Vet World. 2011:4:423-8.

6. Waturangi DE, Pradita N, Linarta J, Banerjee S. Prevalence and molecular characterization of Vibrio cholerae from ice and beverages sold in Jakarta, Indonesia, using most probable number and multiplex PCR. J Food Prot. 2012;75:651-9.
7. Correa NE, Lauriano CM, McGee R, Klose KE. Phosphorylation of the flagellar regulatory protein $\mathrm{FlrC}$ is necessary for Vibrio cholerae motility and enhanced colonization. Mol Microbiol. 2000;35:743-55.

8. Rivera ING, Chun J, Huq A, Sack RB, Colwell RR. Genotypes associated with virulence in environmental isolates of Vibrio cholerae. Appl Environ Microbiol. 2001;67:2421-9.

9. Pinto AD, Ciccarese G, Tantillo G, Catalano D, Forte VT. A collagenase-targeted multiplex PCR assay for identification of Vibrio alginolyticus, Vibrio cholerae, and Vibrio parahaemolyticus. J Food Prot. 2005;68:150-3.

10. Khuntia HK, Pal BB, Chhotray GR. Quadruplex PCR for simultaneous detection of serotype, biotype, toxigenic potential, and central regulating factor of Vibrio cholerae. J Clin Microbiol. 2008;46:2399-401.

11. Singh DV, Isac SR, Colwell RR. Development of a hexaplex PCR assay for rapid detection of virulence and regulatory genes in Vibrio cholerae and Vibrio mimicus. J Clin Microbiol. 2002;40:4321-4.

12. Clinical and Laboratory Standards Institute [[CLSI]. Disc Diffusion Suplemental Tables; 2013. http://www.oxoid.com/pdf/uk/2013-CLSIFDA-table-update.pdf. Accessed 2 July 2013.

13. Matson JS, Withey JH, Dirita VJ. Regulatory networks controlling Vibrio cholerae virulence gene expression. Infect Immun. 2007:75:5542-9.

14. Agtini MD, et al. The burden of diarrhoea, shigellosis, and cholera in North Jakarta, Indonesia: findings from 24 months surveillance. BMC Infect Dis. 2007;5:89.

15. Bik EM, Bunschoten AE, Gouw RD, Mooi FR. Genesis of the novel epidemic Vibrio cholerae 0139 strains: evidence for horizontal transfer of genes involved in polysaccharide synthesis. J EMBO. 1995;14:209-16.

16. Ramamurthy T, Bag PK, Pal A, Bhattacharya SK, Shimada T, Takeda T, Karasawa T, Kurasono H, Takeda Y, Nair GB. Virulence patterns of Vibrio cholerae non-O1 strains isolated from hospitalized patients with acute diarrhea in Calcuta, India. J Med Microbiol. 1993;39:310-7.

17. Covacci A, Falkow S, Berg DE, Rappuoli R. Did the inheritance of a pathogenicity island modify the virulence of Helicobacter pyroli. J Microbiol. 1997;5:205-8.

\section{Publisher's Note}

Springer Nature remains neutral with regard to jurisdictional claims in published maps and institutional affiliations.
Ready to submit your research? Choose BMC and benefit from:

- fast, convenient online submission

- thorough peer review by experienced researchers in your field

- rapid publication on acceptance

- support for research data, including large and complex data types

- gold Open Access which fosters wider collaboration and increased citations

- maximum visibility for your research: over $100 \mathrm{M}$ website views per year

At BMC, research is always in progress.

Learn more biomedcentral.com/submissions 